\title{
Los repartimientos del «servicio y medio servicio» de los judíos de Castilla de 1484, 1485, 1490 y 1491
}

\author{
Gonzalo ViñuALES FERREIRO \\ UNED, Madrid
}

Hace ahora treinta años, Miguel Ángel Ladero Quesada hizo públicas las contribuciones de las juderías de Castilla en concepto de «servicio y medio servicio» a la hacienda regia en los años 1450, 1453, 1464,1472 y $1479{ }^{1}$. Por las mismas fechas, Francisco Cantera Burgos y Carlos Carrete Parrondo ofrecieron una magnífica edición del repartimiento del «servicio y medio servicio» de los años de 1472 y $1474^{2}$. Varios años antes, Luis Suárez Fernández había sacado a la luz la contribución correspondiente al año 1482, acompañada de las que se referían al pago de «los castellanos de oro», ya para los años de la guerra de Granada de 1485, 1486, 1488, 1489, 1490 y 1491 .

En las páginas que siguen se completa la información ofrecida en los trabajos de los mencionados estudiosos con las listas que hemos encontrado en la sección de Escribanía Mayor de Rentas, del Archivo General de Simancas, y que corresponden a las contribuciones de las aljamas y juderías de Castilla, en concepto de «servicio y medio servicio», en los años de 1484, 1485, 1490 y 1491.

1 M. A. LADERo QUeSADA, «Las juderías de Castilla según algunos 'servicios' fiscales del siglo XV», Sefarad 31 (1971) págs. 249-264.

${ }^{2}$ F. CANTERA BuRgos y C. CARRETE PARRONDO, «Los repartimientos de Rabí Jaco Aben Núñez», Sefarad 31 (1971) págs. 212-264.

3 L. SuÁREZ FERnÁNDEZ, Documentos acerca de la expulsión de los judíos (Valladolid 1964) pags. 65-72.

Sefarad 62 (2002) págs. 185-206

(C) CSIC

ISSN 037-0894 
$\mathrm{El}$ «servicio y medio servicio» nació como un impuesto de carácter extraordinario y específico para las minorías étnicas de judíos y mudéjares de Castilla. Las arcas reales se embolsaban en cada contribución 600.000 maravedíes: $450.000 \mathrm{mrs}$. correspondientes a los judíos, y 150.000 mrs. recaudados entre los mudéjares. Con el discurrir del tiempo, el tributo se consolidó y pasó a cobrarse anualmente, aunque sin modificar su montante, de manera que su importancia fue disminuyendo a medida que la moneda veía reducido su valor. No obstante, siguió percibiéndose hasta el final de la presencia judía ${ }^{4}$.

Como sucediera en 1479 y 1482, se encomendaron los repartos de 1484, 1485, 1490 y 1491 a don Abraham Seneor, Rabí mayor de las aljamas de los judíos de Castilla. Este impuesto se cobraba mediante el sistema de encabezamiento, lo que exigía un reparto equitativo entre localidades y habitantes, argumento que permitió a Ladero Quesada exponer la posibilidad de realizar un análisis geográfico y demográfico de la población judía de Castilla a fines del siglo XV.

La distribución espacial se nos presenta obvia, al aparecer enunciados todos aquellos núcleos de población que contaban con vecinos hebreos. En relación con las listas publicadas por Ladero, no se observa ninguna alteración significativa en el poblamiento judío en Castilla. Se constata la escasez de núcleos judíos en Galicia, y se confirma la desaparición, a partir de 1485, de muchas de las aljamas andaluzas, como ya había señalado Suárez Fernández. El resto es muy semejante.

Por otra parte, la prudencia nos ha impedido aventurar hipótesis derivadas de una comparación entre las listas correspondientes al reparto del «servicio y medio servicio» y el de «los castellanos de oro». Al ser ambos sufragados por los judíos, y al coincidir las fechas de 1485, 1490 y 1491, las opciones de valorar la realidad judía en Castilla para esta época se duplicaban. Sin embargo, esta realidad se nos iba a mostrar muy poco clarificadora, pues no existe una relación objetiva

\footnotetext{
${ }^{4}$ En el legajo (en adelante, Leg.) número 51, de la sección de Escribanía Mayor de Rentas (en adelante, EMR), del Archivo General de Simancas (en adelante, AGS), encontramos un documento que hace referencia al cargo del cobro del «servicio y medio servicio» de los moros y de los judios de Castilla correspondiente al año 1492, por lo que podemos considerar que es muy posible que este impuesto se cobrara incluso el mismo año de la expulsión.
} 
entre las cantidades que paga una aljama en virtud de cada tributo, ni ninguna correlación entre lo abonado por unas aljamas y por otras.

Sirva de muestra el siguiente ejemplo. La aljama judía de Maqueda contribuye en el «servicio y medio servicio» con 20.000 maravedíes en 1490 y 1491. Es la comunidad hebrea de Castilla que más paga. Ahora bien, en concepto de «castellanos de oro», sólo tributa con 50.000 maravedíes, mientras que Segovia, que había contribuido con 11.000 maravedíes en el «servicio y medio servicio», iba a pagar, en esas mismas fechas, 151.640 y 140.000 maravedíes respectivamente para los gastos de la guerra de Granada.

Así pues, parece razonable afirmar que la contribución del «servicio y medio servicio», aunque se sigue recaudando hasta la expulsión de 1492, tiene una importancia reducida frente a un impuesto más actual y específico, como el de «los castellanos de oro», donde se pone más claramente de manifiesto la realidad objetiva de las comunidades hebreas de Castilla al terminar el siglo XV.

Pese a todo, consideramos de indudable interés dar a conocer las listas contributivas que figuran seguidamente.

SERVICIO Y MEDIO SERVICIO DE LOS JUDÍOS DE CASTILLA $(1484,1485$, 1490 Y 1491)

[Nota: Hemos reflejado la cantidad en cursiva en aquellas ocasiones en las que la documentación se refiere a la localidad como aljama]

\begin{tabular}{|l|l|c|c|c|c|}
\hline \multicolumn{7}{|c|}{ LOCALIDAD } & $\mathbf{1 . 4 8 4}^{5}$ & $\mathbf{1 . 4 8 5}^{6}$ & $\mathbf{1 . 4 9 0}^{7}$ & $\mathbf{1 . 4 9 1}^{\mathbf{}}$ \\
\hline \multicolumn{6}{|c|}{ Obispado de Burgos } \\
\hline 1 & Burgos & $700^{9}$ & $700^{10}$ & $700^{\prime \prime}$ & $700^{12}$ \\
\hline
\end{tabular}

${ }^{5}$ AGS. EMR. Leg. 36. Pliegos 150-153.

${ }^{6}$ AGS. EMR. Leg. 39. Pliegos 488-489.

7 AGS. EMR. Leg. 48. Pliegos 237-242.

8 AGS. EMR. Leg. 50. Pliegos 267-269.

9 Con Ubierna y Covarrubias (provincia [en adelante, prov.] de Burgos).

${ }^{10}$ Con Ubierna (prov. de Burgos).

1 Con Ubierna y Covarrubias (prov. de Burgos).

12 Idem 


\begin{tabular}{|c|l|c|c|c|c|}
\hline 2 & Cabia & 200 & 200 & 225 & 200 \\
\hline 3 & Herrera (de Pisuerga) & 3.000 & $3.000^{13}$ & $3.000^{14}$ & $3.000^{15}$ \\
\hline 4 & Aguilar (de Campoo) & $1.000^{16}$ & $1.000^{15}$ & $1.000^{17}$ & $1.000^{18}$ \\
\hline 5 & Cervera de Vallejera & 1.000 & 1.000 & 500 & 500 \\
\hline 6 & Medina de Pomar & $2.500^{19}$ & $2.500^{20}$ & $2.500^{21}$ & $3.000^{22}$ \\
\hline 7 & Oña & 400 & 400 & 400 & 400 \\
\hline 8 & Poza & 700 & 700 & 700 & 700 \\
\hline 9 & Frías & 200 & 200 & 900 & 900 \\
\hline 10 & Valmaseda & 1.500 & $1.000^{23}$ & - & - \\
\hline 11 & Miranda de Ebro & $2.000^{24}$ & $2.000^{25}$ & $1.000^{26}$ & $1.000^{27}$ \\
\hline 12 & Berantevilla & - & 500 & $555^{23}$ & $555^{23}$ \\
\hline 13 & Salinas de Añana & $500^{28}$ & $500^{29}$ & $500^{30}$ & $500^{31}$ \\
\hline
\end{tabular}

${ }^{13}$ Con Osorno (prov. de Palencia).

${ }_{14}$ Idem.

${ }^{15}$ Idem.

${ }_{17}^{16}$ Con Reinosa (prov. de Santander).

${ }_{17}$ Idem.

${ }_{18}$ Idem

19 Con los que con ellos suelen pechar.

${ }^{20}$ Idem.

${ }^{21}$ Con Arroyuelo, Espinosa (prov. de Burgos) y Ampuero (prov. de Santander).

${ }_{22}^{22}$ Idem.

${ }^{23}$ Con Laredo (prov. de Santander)

${ }^{24}$ Con los que con ellos suelen pechar.

${ }^{25}$ Con Fontecha y Ocio (prov. de Álava)

${ }^{26}$ Con Fontecha, Ocio, Estavillo (prov. de Álava) y Viana (prov. de Navarra).

27 Idem.

${ }^{28}$ Con los que con ellos suelen pechar.

${ }^{29}$ Idem.

${ }^{30}$ Con Urueña, Caicedo, Puentelarra y Antezana (prov. de Álava).

31 Idem. 


\begin{tabular}{|c|l|c|c|c|c|}
\hline 14 & Briviesca & $1.500^{32}$ & $1.500^{33}$ & $1.500^{34}$ & $1.500^{35}$ \\
\hline 15 & Pancorbo & $800^{36}$ & $800^{37}$ & $1.500^{38}$ & $1.500^{39}$ \\
\hline 16 & Santa Gadea & 1.500 & 1.500 & 1.200 & 1.200 \\
\hline 17 & Castrojeriz & 600 & 600 & 400 & 400 \\
\hline 18 & Astudillo & 800 & 800 & 1.000 & 1.000 \\
\hline 19 & Belorado & $2.400^{40}$ & $2.400^{41}$ & $3.400^{42}$ & $3.400^{43}$ \\
\hline 20 & Valdezcaray & - & - & $3.750^{44}$ & 3.75045 \\
\hline 21 & Cerezo & 200 & 200 & 300 & 300 \\
\hline 22 & Redecilla (del Camino) & 1.200 & 1.200 & 800 & 800 \\
\hline 23 & Grañón & 2.000 & 500 & 500 & 500 \\
\hline 24 & Villadiego & 1.000 & 1.000 & 1.000 & 1.000 \\
\hline & Total del obispado & 25.700 & 24.200 & 27.330 & 27.805 \\
\hline
\end{tabular}

32 Con los que con ellos suelen pechar.

33 Con Salas, Monasterio de Rodilla, Quintanaopio y los lugares de la merindad de Bureba que no se nombran en otra parte (prov. de Burgos).

${ }^{34}$ Idem.

35 Idem.

36 Con Busto (prov. de Burgos)

37 Idem.

38 Idem.

39 Idem.

${ }^{40}$ Con los que con ellos suelen pechar.

41 Con Ojocastro, Valgañón, Villaharta y Quintanar (Villarta-Quintana) (prov. de La Rioja), ¿Velmán?, Val de San Vicente (San Vicente del Valle), La Fresneda (Fresneda de la Sierra Tirón), Villafranca y sus aldeas, Villanasur, Santa María de la Invierna (Santa María del Invierno), Piedrahita, y con los que con ellos pechan (prov. de Burgos).

42 Con Villafranca, Quintanares, Villa de Pun, Val de San Vicente (vid. supra), La Fresneda (vid. supra), Villanasur, Santa María de la Invierna (vid. supra), Piedrahita, Milanos, Arlanzón, Quintanilla de San García, Galarde, Olmos, Ajese (Agés), Valluércanes, y Avellanosa (prov. de Burgos), Villaharta y sus aldeas (vid. supra) (prov. de La Rioja).

43 Idem.

44 Con Ojocastro, Valgañón, Santurde y Santurdejo (prov. de La Rioja).

45 Idem. 


\begin{tabular}{|c|l|r|r|r|r|}
\hline \multicolumn{5}{|c|}{ Obispado de Calahorra } \\
\hline 25 & Calahorra & 1.000 & 1.000 & 1.000 & 1.000 \\
\hline 26 & Jubera & $400^{46}$ & $400^{47}$ & 400 & 400 \\
\hline 27 & Arnedo & $1.500^{48}$ & $1.500^{49}$ & $2.500^{50}$ & $2.500^{51}$ \\
\hline 28 & Herce & 600 & 300 & 300 & 300 \\
\hline 29 & Préjano & - & 400 & 450 & 450 \\
\hline 30 & San Pedro de Yanguas & 500 & 500 & 1.500 & 1.500 \\
\hline 31 & Alfaro & 700 & 700 & 700 & 700 \\
\hline 32 & $\begin{array}{l}\text { Cervera de Río } \\
\text { Alhama }\end{array}$ & 2.300 & 2.500 & 3.750 & 3.750 \\
\hline 33 & Cornago & 1.000 & 1.000 & 1.000 & 1.000 \\
\hline 34 & Salvatierra de Álava & 1.600 & $1.600^{52}$ & 1.500 & 1.500 \\
\hline 35 & $\begin{array}{l}\text { Santa Cruz de } \\
\text { Campezo }\end{array}$ & 300 & 300 & 450 & 450 \\
\hline 36 & Treviño & 1.700 & 1.700 & 1.700 & 1.700 \\
\hline 37 & Vitoria & $3.000^{53}$ & $3.000^{54}$ & 4.00055 & 3.00056 \\
\hline
\end{tabular}

${ }^{46}$ Con Enciso (prov. de La Rioja).

${ }^{47}$ Idem.

${ }^{48}$ Con Arnedillo (prov. de La Rioja).

${ }^{49}$ Con Arnedillo y Ausejo (prov. de La Rioja).

${ }^{50}$ Idem.

${ }^{51}$ Idem.

52 Con Antoñana (prov. de Álava).

${ }^{53}$ Con los que con ellos pechan.

${ }^{54}$ Con Yrraraçabal, Evargüena, Ozaeta, Segura, Guevara, Arlanda, Mondragón (prov. de Guipúzcoa) y Orduña (prov. de Vizcaya).

${ }^{55}$ Con Yrraraçabal, Evargüena, Ozaeta, Segura, Guevara, Arlanda, Mondragón (prov. de Guipúzcoa) y Orduña (prov. de Vizcaya).

${ }^{56}$ Con Yrraraçabal, Evargüena, Ozaeta, Segura, Guevara, Arlanda, Mondragón (prov. de Guipúzcoa) y Orduña (prov. de Vizcaya). 


\begin{tabular}{|c|l|r|r|r|r|}
\hline 38 & Haro & 1.100 & $1.100^{57}$ & $1.650^{58}$ & $1.650^{59}$ \\
\hline 39 & Leyva & 500 & 500 & 350 & 350 \\
\hline 40 & Briones & $1.000^{60}$ & 1.000 & 1.000 & 1.000 \\
\hline 41 & La Guardia & 1.000 & 1.000 & 1.500 & 1.500 \\
\hline 42 & Bañares & $1.000^{61}$ & $1.000^{62}$ & 500 & 500 \\
\hline 43 & Labastida & 700 & 700 & 700 & 700 \\
\hline 44 & Salinillas & 500 & $500^{63}$ & 700 & 700 \\
\hline 45 & Entrena & - & - & 300 & 300 \\
\hline 46 & Treviana & 400 & 400 & 450 & 450 \\
\hline 47 & Navarrete & $2.500^{64}$ & $2.500^{65}$ & 1.500 & 1.500 \\
\hline 48 & Nájera & $600^{66}$ & $600^{67}$ & 500 & 500 \\
\hline 49 & S. Millán de la Cogolla & - & - & 550 & 550 \\
\hline 50 & Logroño & 400 & 400 & 400 & 400 \\
\hline & Total obispado & 24.300 & 24.600 & 29.350 & 28.350 \\
\hline
\end{tabular}

\begin{tabular}{|l|l|r|r|r|r|}
\hline \multicolumn{7}{|c|}{ Obispado de Palencia } \\
\hline 51 & Palencia & 1.700 & 1.700 & 600 & 600 \\
\hline
\end{tabular}

${ }^{57}$ Con Çeratón (Zarratón), Saja (Sajazarra), Fonzaleche (prov. de La Rioja) y Peñacerrada (prov. de Álava).

58 Idem.

59 Idem.

${ }^{60}$ Con San Vicente (San Vicente de la Sonsierra) (prov. de La Rioja).

61 Con Villar (prov. de La Rioja).

62 Idem.

63 Con Buradón (prov. de Álava).

${ }^{64}$ Con Huércanos y Las Arenzanas (Arenzana de Arriba y Arenzana de Abajo) (prov. de La Rioja).

${ }^{65}$ Con Huércanos, Las Arenzanas (vid. supra) y Entrena (prov. de La Rioja).

${ }^{66}$ Con los que con ellos pechan.

${ }^{67}$ Con San Millán de la Cogolla y Cañas (prov. de La Rioja). 


\begin{tabular}{|c|c|c|c|c|c|}
\hline 52 & Palenzuela & 200 & 200 & 200 & 200 \\
\hline 53 & Dueñas & 2.400 & 2.400 & 1.400 & 1.400 \\
\hline 54 & Cevico de la Torre & - & - & 300 & 300 \\
\hline 55 & Sahagún & 1.500 & 1.500 & 1.200 & 1.200 \\
\hline 56 & Villalón (de Campos) & 6.000 & 6.000 & 5.000 & 5.000 \\
\hline 57 & Cea & 2.000 & 2.000 & 1.000 & 1.000 \\
\hline 58 & Grajal & 2.000 & 1.000 & 800 & 800 \\
\hline 59 & Melgar de Suso & $1.500^{68}$ & $1.000^{69}$ & 700 & 700 \\
\hline 60 & Villada & - & - & 375 & 375 \\
\hline 61 & Saldaña & 2.000 & 2.000 & 3.000 & 3.000 \\
\hline 62 & Almanza & $1.500^{70}$ & 1.500 & 1.200 & 1.200 \\
\hline 63 & Frómista & $16.000^{71}$ & $3.000^{72}$ & $3.000^{73}$ & $3.000^{74}$ \\
\hline 64 & Tordesillas & 500 & 1.000 & 1.000 & 1.000 \\
\hline 65 & Ampudia & 2.000 & 3.000 & 3.000 & 1.500 \\
\hline 66 & Torre de Mormojón & $2.000^{75}$ & $2.000^{76}$ & 2.500 & 2.500 \\
\hline 67 & Cisneros & - & - & 300 & 300 \\
\hline 68 & Amusco & 2.000 & 2.000 & 2.000 & 2.000 \\
\hline 69 & Carrión & 1.000 & 1.000 & 1.000 & 1.000 \\
\hline
\end{tabular}

${ }^{68}$ Con Melgar de Yuso (Melgar de Arriba) (prov. de Valladolid).

69 Idem.

${ }^{70}$ Con Valverde de Arcayos (prov. de Palencia).

${ }^{71}$ Con Piña (Piña de Campos), (prov. de Palencia)

${ }^{72}$ Idem.

${ }^{73}$ Con Piña (vid. supra), Avia (Abia de Torres), Revenga (Revenga de Campos), Castrillo y Villovela (prov. de Palencia).

${ }_{74}$ Idem.

${ }^{75}$ Con Becerril (Becerril de Campos) y Paredes de Nava (prov. de Palencia).

${ }^{76}$ Idem. 


\begin{tabular}{|c|l|r|r|r|r|}
\hline 70 & Monzón & - & 800 & 540 & 540 \\
\hline 71 & Valladolid & $5.500^{77}$ & $5.500^{78}$ & $3.500^{79}$ & $3.500^{80}$ \\
\hline 72 & Aguilar de Campos & $5.000^{81}$ & 2.500 & 2.000 & 2.000 \\
\hline 73 & Medina de Rioseco & - & $2.500^{82}$ & $2.650^{83}$ & $2.650^{84}$ \\
\hline 74 & Villabrágima & - & - & 1.000 & 1.000 \\
\hline 75 & Torrelobatón & 4.400 & 3.000 & $3.300^{85}$ & $3.300^{86}$ \\
\hline 76 & Belver & 1.000 & 1.000 & 500 & 500 \\
\hline 77 & Cuenca de Villalón & 2.500 & 2.500 & 1.000 & 1.000 \\
\hline 78 & Urueña & & & & $1.000^{89}$ \\
\hline 79 & Palazuelo de Vedija & - & $500^{90}$ & - & $-1.000^{88}$ \\
\hline 80 & Villagarcía & 700 & 700 & 450 & 450 \\
\hline 81 & Tordehumos & 2.000 & 2.000 & 800 & 800 \\
\hline 82 & Villafrechós & 300 & 300 & 300 & 300 \\
\hline & Total del obispado & 68.200 & 55.100 & 45.615 & 44.115 \\
\hline
\end{tabular}

${ }_{77}^{77}$ Con Zaratán, Portillo, Cigales y Mucientes (prov. de Valladolid).

78 Idem.

79 Idem

80 Idem

81 Con los que con ellos pechan.

82 Con Villabrágima, Tamares (Tamariz de Campos), Moral de la Reina, Bolaños (Bolaños de Campos) y Vecilla (Becilla de Valderaduey) (prov. de Valladolid).

${ }^{83}$ Con Tamares (vid. supra), Moral de la Reina, Bolaños (vid. supra) y Vezilla (vid. supra) (prov. de Valladolid).

84 Idem.

${ }^{85}$ Con Wamba y Peñaflor (Peñaflor de Hornija) (prov. de Valladolid).

86 Idem.

${ }^{87}$ Con los que con ellos pechan.

${ }^{88}$ Con Villardefrades (prov. de Zamora).

Idem.

${ }^{90}$ Con Valdenebro (de los Valles) y Montealegre (de Campos) (prov. de Valladolid). 


\begin{tabular}{|c|l|c|c|c|c|}
\hline \multicolumn{6}{|c|}{ Obispado de Osma ${ }^{91}$} \\
\hline 83 & Soria & 8.000 & 8.000 & 8.000 & 8.000 \\
\hline 84 & Calatañazor & 1.000 & 1.000 & 1.000 & 1.000 \\
\hline 85 & Burgo de Osma & 300 & 300 & 300 & 300 \\
\hline 86 & Serón & 80092 & $800^{93}$ & 800 & 800 \\
\hline 87 & Roa & 1.500 & 1.500 & 800 & 800 \\
\hline 88 & Aranda & - & - & 1.000 & 2.000 \\
\hline 89 & Peñaranda & 1.500 & 1.500 & 1.000 & 1.000 \\
\hline 90 & Gumiel de Mercado & 70094 & $700^{95}$ & 700 & 700 \\
\hline 91 & Ágreda & 1.000 & 1.000 & 1.000 & 1.000 \\
\hline 92 & Curiel & 200 & 20096 & - & - \\
\hline 93 & Coruña (del Conde) & 4.000 & 4.000 & 2.250 & 2.250 \\
\hline & Total del obispado & 19.500 & 19.500 & 16.850 & 17.850 \\
\hline
\end{tabular}

\begin{tabular}{|c|l|c|c|c|c|}
\hline \multicolumn{6}{|c|}{ Obispado de Sigüenza } \\
\hline 94 & Sigüenza & $1.500^{97}$ & $1.500^{98}$ & $1.000^{99}$ & $1.000^{100}$ \\
\hline 95 & Medinaceli & 1.500 & 1.500 & 1.750 & 1.750 \\
\hline 96 & Atienza & 1.200 & 1.200 & 1.300 & 2.000 \\
\hline
\end{tabular}

${ }^{91}$ F. CANTERA Burgos, «Juderías medievales en la provincia de Soria», en Homenaje a Fray Justo Pérez de Urbel, OSB, vol. I (Silos 1976) págs. 445-482.

${ }_{92}$ Con los que con ellos pechan.

${ }_{93}$ Con Monteagudo (de las Vicarías) (prov. de Soria).

${ }^{94}$ Con Gumiel de Hizán (prov. de Burgos).

${ }^{95}$ Idem.

${ }^{96}$ Con Enzinillas (¿prov. de Segovia?).

${ }^{97}$ Con Cifuentes, Baides y Aldea Seca (prov. de Guadalajara).

${ }^{98}$ Idem.

99 Idem.

100 Idem. 


\begin{tabular}{|c|l|c|c|c|c|}
\hline 97 & Caracena & $1.000^{101}$ & $1.000^{102}$ & 600 & 600 \\
\hline 98 & Ayllón & $2.200^{103}$ & $2.200^{104}$ & 2.200 & 2.200 \\
\hline 99 & San Esteban de Gormaz & 1.050 & 1.050 & 1.200 & 1.200 \\
\hline 100 & Almazán & $4.500^{105}$ & $4.500^{106}$ & 4.500 & 4.500 \\
\hline 101 & Berlanga & 500 & 500 & 1.000 & 1.000 \\
\hline 102 & Fuentepinilla & 250 & $250^{107}$ & 450 & 450 \\
\hline & Total del obispado & 13.700 & 13.700 & 14.000 & 14.700 \\
\hline
\end{tabular}

\begin{tabular}{|c|l|c|c|c|c|}
\hline \multicolumn{7}{|c|}{ Obispado de Segovia } \\
\hline 103 & Segovia & $11.000^{108}$ & $11.000^{109}$ & $11.000^{110}$ & $11.000^{111}$ \\
\hline 104 & Coca & $1.000^{112}$ & $1.000^{113}$ & 1.000 & 1.000 \\
\hline 105 & Cuéllar & $2.500^{113}$ & $2.500^{1}$ & 2.500 & 2.500 \\
\hline 106 & Peñafiel & $3.000^{114}$ & $3.000^{115}$ & 4.000 & 4.000 \\
\hline 107 & Fuentidueña & $1.000^{116}$ & $1.000^{117}$ & 1.000 & 1.000 \\
\hline
\end{tabular}

${ }^{101}$ Con Negrales (prov. de Soria).

102 Idem.

103 Con Maderuelo y Riaza (prov. de Segovia).

104 Idem.

${ }^{105}$ Con Morón (prov. de Soria).

106 Idem.

107 Con Tajueco (prov. de Soria).

108 Con sus ayudas.

109 Y sus ayudas.

${ }^{110}$ Con Chinchón (prov. de Madrid), Turégano y Sepúlveda (prov. de Segovia).

111 Idem.

112 Con Lagunillas (prov. de Segovia).

113 Idem.

${ }^{114}$ Con Pinel de Suso y Pinel de Yuso (Piñel de Abajo, y Piñel de Arriba) (prov. de Valladolid).

115 Idem.

${ }_{117}$ Con los que con ellos pechan.

117 Con Lagunillas (prov. de Segovia). 


\begin{tabular}{|c|l|c|c|c|c|}
\hline 108 & Pedraza & $2.000^{118}$ & $2.000^{119}$ & 1.000 & 1.000 \\
\hline 109 & Sepúlveda & - & 200 & - & - \\
\hline & Total del obispado & 20.500 & 20.700 & 20.500 & 20.500 \\
\hline
\end{tabular}

\begin{tabular}{|c|l|c|c|c|c|}
\hline \multicolumn{5}{|c|}{ Obispado de Ávila } \\
\hline 110 & Ávila & 8.000 & 8.000 & 5.000 & 5.000 \\
\hline 111 & Villanueva de Gómez & - & $300^{120}$ & 300 & 200 \\
\hline 112 & Villatoro & 1.000 & 1.000 & 1.000 & 1.000 \\
\hline 113 & Piedrahita & 3.500 & 3.500 & 2.500 & 2.500 \\
\hline 114 & Barco de Ávila & 6.000 & 6.000 & 5.300 & 5.300 \\
\hline 115 & Olmedo & 500 & 500 & 500 & 500 \\
\hline 116 & Oropesa & 2.500 & 2.500 & 2.500 & 2.500 \\
\hline 117 & Candeleda & 800 & 800 & 600 & 600 \\
\hline 118 & Medina del Campo & 8.000 & 8.000121 & 8.000122 & 8.000123 \\
\hline 119 & La Adrada & 1.400 & 1.400 & 1.400 & 1.400 \\
\hline 120 & Colmenar de Arenas & 2.500 & 2.500 & - & - \\
\hline 121 & Arenas (de San Pedro) & 1.400 & 1.400 & 1.000 & 1.000 \\
\hline 122 & Alaejos & - & - & 250 & 250 \\
\hline 123 & Arévalo & 2.000 & 2.000 & 3.000 & 3.000 \\
\hline
\end{tabular}

118 Con Collado (Collado Hermoso) (prov. de Segovia).

119 Idem.

120 Con Bondo (El Bohodón) (prov. de Ávila).

${ }^{121}$ Con Fuente el Sol, Bovadilla (Bobadilla del Campo) (prov. de Valladolid).

122 Con Fuente el Sol, Bovadilla (vid. supra) y Fresno (Fresno el Viejo) (prov. de Valladolid).

123 Idem. 


\begin{tabular}{|c|l|c|c|c|c|}
\hline 124 & Madrigal & 4.000 & 4.000 & 4.000 & 4.000 \\
\hline 125 & $\begin{array}{l}\text { Peñaranda de Álvaro } \\
\text { de Bracamonte } 124\end{array}$ & $1.000^{125}$ & $1.000^{126}$ & 1.000 & 1.000 \\
\hline 126 & Mombeltrán & - & - & 2.500 & 2.500 \\
\hline 127 & Bonilla de la sierra & 3.500 & 3.500 & 3.500 & 3.500 \\
\hline 128 & Navamorcuende & $2.000^{127}$ & $2.000^{128}$ & 1.200 & 1.200 \\
\hline 129 & Villafranca & 1.500 & 1.500 & 400 & 400 \\
\hline 130 & $\begin{array}{l}\text { Las Navas de Pedro de } \\
\text { Ávila 129 }\end{array}$ & 500 & 300 & 600 & 300 \\
\hline & Total del obispado & 50.100 & 50.200 & 44.550 & 44.150 \\
\hline
\end{tabular}

\begin{tabular}{|c|l|c|c|c|c|}
\hline \multicolumn{7}{|c|}{ Obispado de Salamanca ciudad Rodrigo ${ }^{130}$} \\
\hline 131 & Salamanca & $4.800^{131}$ & $4.800^{132}$ & $3.800^{133}$ & $3.800^{134}$ \\
\hline 132 & Monleón & - & - & 500 & 500 \\
\hline 133 & Ciudad Rodrigo & 2.000 & 2.000 & $3.000^{135}$ & $3.500^{136}$ \\
\hline
\end{tabular}

124 Peñaranda de Bracamonte (prov. de Salamanca).

125 Con Paradinas (Paradinas de San Juan) (prov. de Salamanca).

126 Idem.

127 Con Pajares, Cardiel (Cardiel de los Montes) y San Román (San Román de los Montes) (prov. de Toledo).

128 Idem.

129 Las Navas del Marqués (prov. de Ávila).

130 C. Carrete Parrondo, Fontes Iudaeorum Regni Castellae I: Provincia de Salamanca (Salamanca. 1981) págs. 145-150.

131 Con los que con ellos pechan.

132 Con sus ayudas.

133 Con Tamames, Texeda y La Hornada (prov. de Salamanca).

134 Idem.

135 Con La Hinojosa (prov. de Salamanca).

136 Idem. 


\begin{tabular}{|c|l|c|c|c|c|}
\hline 134 & Alba de Tormes & 2.400 & $2.400^{137}$ & $2.400^{138}$ & $2.400^{139}$ \\
\hline 135 & Ledesma & 1.800 & 1.800 & 1.800 & 1.800 \\
\hline 136 & Salvatierra de Tormes & 2.000 & 2.000 & 2.000 & 1.000 \\
\hline 137 & Miranda del Castañar & 2.000 & 1.500 & 1.400 & 1.400 \\
\hline 138 & Cantalapiedra & 500 & 500 & $450^{140}$ & $400^{141}$ \\
\hline 139 & La Hinojosa & - & $300^{142}$ & - & - \\
\hline & Total del obispado & 15.500 & 15.300 & 15.350 & 14.800 \\
\hline
\end{tabular}

\begin{tabular}{|c|l|c|c|c|c|}
\hline \multicolumn{5}{|c|}{ Obispado de Zamora } \\
\hline 140 & Zamora $^{143}$ & $6.500^{144}$ & $6.500^{145}$ & $6.500^{146}$ & $6.500^{147}$ \\
\hline 141 & Toro & $1.500^{148}$ & $1.500^{149}$ & 1.500 & 1.500 \\
\hline 142 & Fuentesaúco ${ }^{150}$ & 500 & $500^{151}$ & $900^{152}$ & $900^{153}$ \\
\hline 143 & Alcañices & 500 & 500 & 700 & 700 \\
\hline & Total del obispado & 9.000 & 9.000 & 9.600 & 9.600 \\
\hline
\end{tabular}

137 Con Villoria (prov. de Salamanca).

138 Idem.

139 Idem.

${ }^{140}$ Con Torrecilla (Torrecilla de la Orden) (prov. de Salamanca).

141 Idem.

142 Con San Martín de Trebe (San Martín de Trevejo) (prov. de Cáceres), Fuenteguinaldo y Robladillo (prov. de Salamanca).

${ }^{143}$ M. F. GARCíA CASAR, El pasado judío de Zamora (Valladolid 1992) pág. 115.

${ }^{144}$ Con los que con ellos suelen pechar.

145 Con los lugares de sus ayudas.

${ }^{146}$ Con Castrotorafe, Fermoselle y San Pedro de Latarce (prov. de Zamora).

147 Idem.

148 Con Castronuño y San Román de Hornija (prov. de Zamora).

149 Con Castronuño, y San Román de Hornija, sin La Mota (prov. de Zamora).

150 C, CARRETE PARRONDO, «Sinagogas e impuestos fiscales de dos comunidades zamoranas: Fermoselle y Fuentesaúco», Helmántica 34 (1983) págs. 109-113.

151 Con Venialbo y El Maderal (prov. de Zamora).

${ }_{152}$ Con Venialbo, El Maderal y Fuentelapeña (prov. de Zamora).

153 Idem. 


\begin{tabular}{|c|l|c|c|c|c|}
\hline \multicolumn{5}{|c|}{ Obispado de León y Astorga ${ }^{154}$} \\
\hline 144 & León & 3.500 & 3.500 & 3.500 & 3.500 \\
\hline 145 & Laguna de Negrillos & 3.000 & $1.500^{155}$ & 1.500 & 1.500 \\
\hline 146 & Villamañán & 1.500 & $1.500^{156}$ & 500 & 500 \\
\hline 147 & Valencia de León ${ }^{157}$ & 2.000 & 2.500 & 2.000 & 2.000 \\
\hline 148 & Mansilla (de las Mulas) & 3.000 & 3.000 & 1.200 & 1.200 \\
\hline 149 & Mayorga & 8.000 & 8.000 & 8.000 & 8.000 \\
\hline 150 & Villalpando & 3.000 & 4.000 & 4.500 & 3.000 \\
\hline 151 & Valderas & 3.000 & $4.000^{158}$ & 3.000 & 3.000 \\
\hline 152 & Castroverde & 1.000 & 1.000 & 825 & 825 \\
\hline 153 & Astorga & 1.000 & 1.000 & 1.375 & 1.375 \\
\hline 154 & Benavides & 1.000 & 1.000 & 1.000 & 1.000 \\
\hline 155 & Palacios de Valduerna & $500^{159}$ & $500^{160}$ & - & - \\
\hline 156 & Ponferrada & $5.500^{161}$ & $5.000^{162}$ & 2.000 & 2.000 \\
\hline 157 & Benavente & 5.500 & 5.500 & 1.000 & 1.000 \\
\hline
\end{tabular}

154 F. CANTERA Burgos, «Juderías medievales de la provincia de León», Archivos Leoneses 28/55-56 (1974) págs. 85-155.

155 Con Pobladura del Valle (prov. de León).

156 Con El ¿Moçolvy? (¿prov. de León?).

157 Valencia de Don Juan (prov. de León).

158 Con Villalobos y Barcial de la Loma (prov. de León).

${ }^{159}$ Con La Bañeza (prov. de León).

160 Idem.

161 Con Los Barrios de Salas, Bembibre, Cacabelos y Villafranca de Valcárcel (prov. de León).

162 Idem. 


\begin{tabular}{|c|l|c|c|c|c|}
\hline 158 & La Coruña & $500^{163}$ & 500 & $500^{164}$ & $500^{165}$ \\
\hline 159 & Orense & $1.000^{166}$ & $1.000^{167}$ & $1.000^{168}$ & $1.000^{169}$ \\
\hline 160 & San Felices de los Gallegos & - & 2.000 & 3.000 & 3.000 \\
\hline 161 & Bayona & 600 & $600^{170}$ & - & - \\
\hline & Total del obispado & 43.600 & 46.100 & 34.900 & 33.400 \\
\hline
\end{tabular}

\begin{tabular}{|c|l|c|c|c|c|}
\hline \multicolumn{6}{|c|}{ Arzobispado de Toledo ${ }^{171}$} \\
\hline 162 & Toledo & $6.500^{172}$ & 6.500 & $6.500^{173}$ & $6.500^{174}$ \\
\hline 163 & Torrijos & 1.500 & 750 & 1.000 & 1.000 \\
\hline 164 & $\begin{array}{l}\text { San Martín de } \\
\text { Valdeiglesias } 175\end{array}$ & 1.000 & 1.000 & 2.500 & 2.500 \\
\hline 165 & Talavera de la Reina & 2.500 & 2.500 & 2.500 & 3.500 \\
\hline 166 & El Puente del Arzobispo & 500 & 500 & 2.000 & 2.000 \\
\hline 167 & Pastrana & 800176 & 800 & 800 & 800 \\
\hline
\end{tabular}

163 Con sus ayudas.

164 Con Ribadeo (prov. de Lugo), Betanzos, La Fuente de Omen (Puentedeume) y Ferrol (prov. de La Coruña).

165 Idem.

166 Con los judíos que con ellos pechan.

167 Con sus ayudas.

168 Con Monforte de Lemos (prov. de Lugo), Allariz, y Rivadavia (prov. de Orense).

169 Idem.

170 Con Tuy (prov. de Pontevedra).

171 C. CARRETE PARRONDO, «Tributación económica de las comunidades judías toledanas», Actas del IV Congreso Internacional «Encuentro de las Tres Culturas». Toledo 30 sept. - 2 oct. 1985 (Toledo 1988) págs. 19-34.

${ }^{172}$ Con Gálvez, Novés, Alcázar de Consuegra, y Las Ventas con Peña Aguilera (prov. de Toledo).

173 Idem.

174 Idem.

175 F. CANTERA Burgos, «La judería de San Martín de Valdeiglesias (Madrid)», Sefarad 29 (1969) págs. 217-312.

${ }^{176}$ Con Zorita (prov. de Guadalajara). 


\begin{tabular}{|c|l|c|c|c|c|}
\hline 168 & Mondéjar & 1.000 & 1.000 & 1.500 & 1.500 \\
\hline 169 & Hita & 3.500 & 3.500 & 3.500 & 3.500 \\
\hline 170 & Torrelaguna & 800 & 800 & 800 & 800 \\
\hline 171 & Uceda & 500 & $500^{177}$ & 1.000 & 1.000 \\
\hline 172 & Talamanca & 1.000 & 1.000 & 1.075 & 1.075 \\
\hline 173 & Tamajón & - & - & 540 & 540 \\
\hline 174 & Maqueda & 3.000 & $12.000^{178}$ & 20.000 & 20.000 \\
\hline 175 & Buitrago & 5.000 & 5.000 & 5.000 & 5.000 \\
\hline 176 & Escalona & 2.000 & 2.000 & 1.350 & 1.350 \\
\hline 177 & Santa Olalla & 2.000 & 2.000 & 3.500 & 3.500 \\
\hline 178 & Puebla de Montalbán & 2.000 & 2.000 & 1.500 & 1.500 \\
\hline 179 & Guadalajara & $7.500^{181}$ & $7.000^{182}$ & $4.500^{183}$ & 4.500184 \\
\hline 180 & Brihuega & - & - & 1.500 & 1.500 \\
\hline 181 & Tendilla & 300 & 300 & 300 & 300 \\
\hline 182 & Alcalá de Henares & $5.500^{185}$ & 5.500 & 5.500 & 6.500186 \\
\hline 183 & Cobeña & 500187 & $1.000^{188}$ & 1.800 & 1.800 \\
\hline
\end{tabular}

177 Con Tamajón (prov. de Guadalajara).

178 Con Camarena, y La Torre de Esteban Ambrán (prov. de Toledo).

179 Idem.

180 Idem.

181 Con los que con ella suelen pechar.

182 Con los judíos de sus ayudas.

183 Con Yunquera, Atanzón, Málaga, Malaguilla, Valdeaveruelo y Chiloeches (prov. de Guadalajara).

184 Idem.

185 Con los que con ellos suelen pechar.

186 Con Santorcad (prov. de Madrid).

187 Con Daganzo (prov. de Madrid).

188 Idem. 


\begin{tabular}{|c|c|c|c|c|c|}
\hline 184 & Uclés & 2.500 & 2.500 & $3.500^{189}$ & $3.500^{190}$ \\
\hline 185 & Ocaña & $11.000^{191}$ & $11.000^{192}$ & $11.000^{193}$ & $11.000^{194}$ \\
\hline 186 & Huete & 2.000 & 3.000 & 2.000 & 2.000 \\
\hline 187 & Casarrubios del Monte & 1.000 & 1.000 & 1.000 & 1.000 \\
\hline 188 & Madrid & $1.100^{195}$ & $1.100^{196}$ & 1.200 & 1.200 \\
\hline 189 & Manzanares & - & - & 700 & 700 \\
\hline 190 & Guadarrama & - & - & 700 & 700 \\
\hline 191 & Torrejón de Velasco & - & 1.000 & 700 & 700 \\
\hline 192 & Pinto & - & $550^{197}$ & 600198 & $600^{199}$ \\
\hline 193 & Illescas & 1.000 & 1.500 & 1.000 & 1.000 \\
\hline 194 & Murcia & 9.000 & 9.000 . & $7.500^{200}$ & $7.500^{201}$ \\
\hline 195 & Mula & - & 800 & 525 & 500 \\
\hline 196 & Lorca & 2.000 & 2.000 & 2.250 & 2.250 \\
\hline \multirow[t]{2}{*}{197} & Cartagena & 500 & 500 & 825 & 825 \\
\hline & Total del arzobispado & 77.500 & 89.600 & 102.165 & 104.140 \\
\hline
\end{tabular}

${ }^{189}$ Con Quintanar, Tarancón y Belinchón (prov. de Cuenca).

190 Con Quintanar, Tarancón, Belinchón y Montalvo (prov. de Cuenca).

191 Con los que con ellos suelen pechar.

192 Con sus ayudas.

193 Con Yepes, Santa Cruz, La Guardia, Colmenar de Oreja, Lillo y Corral de Almaguer (prov. de Toledo).

194 Idem.

195 Con los que con ellos suelen pechar.

196 Sin Torrejón de Velasco, Pinto y Barajas (prov. de Madrid).

197 Con Barajas (prov. de Madrid).

198 Idem.

199 Idem.

200 Con Molina (prov. de Murcia).

201 Idem. 


\begin{tabular}{|c|l|c|c|c|c|}
\hline \multicolumn{6}{|c|}{ Obispado de Plasencia 202} \\
\hline 198 & Plasencia & $5.000^{203}$ & $5.000^{2}$ & $5.000^{204}$ & $5.000^{205}$ \\
\hline 199 & Cabezuela & $1.500^{206}$ & 1.500 & 1.500 & 1.500 \\
\hline 200 & Jaraiz de la Vera & $2.000^{207}$ & $2.000^{208}$ & 2.500 & 2.500 \\
\hline 201 & Belvis & $500^{209}$ & $1.000^{210}$ & $1.800^{211}$ & 1.800 \\
\hline 202 & Jarandilla de la Vera & $5.000^{212}$ & $5.000^{213}$ & $3.000^{214}$ & $3.000^{215}$ \\
\hline 203 & Valverde de la Vera & $1.000^{215}$ & $1.400^{21}$ & $2.000^{21}$ & 2.000 \\
\hline 204 & Garganta la Olla & $300^{216}$ & $300^{217}$ & - & - \\
\hline 205 & Béjar & $6.000^{218}$ & $6.000^{219}$ & $6.500^{220}$ & $6.500^{221}$ \\
\hline 206 & Medellín & $5.000^{222}$ & $5.000^{21}$ & $5.000^{2}$ & 5.000 \\
\hline
\end{tabular}

202 Y. MORENO KOCH, «Contribuciones económicas de los judíos extremeños en la segunda mitad del siglo XV», en Del candelabro a la encina. Raíces hebreas en Extremadura. Hervás, 16, 17, 18 y 19 de marzo de 1995, eds. F. CORTÉs y L. CASTEllano (Badajoz 1996) págs. 105-124.

${ }^{203}$ Con Galisteo y Aldeanueva del Camino (prov. de Cáceres).

${ }^{204}$ Con Losar (de la Vera) y Aldeanueva (prov. de Cáceres).

205 Idem.

206 Con los que con ellos pechan.

207 Con Cuacos de Yuste (prov. de Cáceres).

208 Idem.

209 Con Val de Juncar (Valdehúncar), Almaraz y Saucedilla (prov. de Cáceres).

210 Con Almaraz (prov. de Cáceres).

211 Idem.

212 Con Tornavacas (prov. de Cáceres).

213 Idem.

214 Idem.

215 Idem.

216 Con Pasarón de la Vera (prov. de Cáceres).

217 Idem.

218 Con Hervás (prov. de Cáceres).

219 Idem.

220 Idem.

221 Idem.

222 Con Villanueva de la Serena y Campanario (prov. de Badajoz). 


\begin{tabular}{|c|l|c|c|c|c|}
\hline 207 & Trujillo & $7.500^{223}$ & 7.500 & $7.500^{224}$ & $7.500^{225}$ \\
\hline 208 & Montemayor & 2.500 & 2.500 & 1.500 & 1.500 \\
\hline 209 & Granadilla & 3.000 & 3.000 & 2.500 & 2.500 \\
\hline 210 & Coria & 2.000 & 2.000 & 5.000 & - \\
\hline 211 & Aldeanueva & $300^{226}$ & $300^{227}$ & - & - \\
\hline 212 & Gata & 1.000 & 1.000 & 975 & 975 \\
\hline 213 & Cáceres & $5.700^{228}$ & 5.700 & 5.500 & 7.000 \\
\hline 214 & Arroyo del Puerco & 800 & 400 & 400 & 400 \\
\hline 215 & Alcántara & 1.000 & 1.000 & 2.250 & 2.250 \\
\hline 216 & Valencia de Alcántara & 1.000 & 1.000 & 1.500 & 1.500 \\
\hline 217 & Alburquerque & 2.000 & 2.000 & 3.500 & 3.000 \\
\hline 218 & Villanueva de la Serena & - & 500 & 600 & 600 \\
\hline 219 & Garrovillas & - & 500 & 450 & 450 \\
\hline & Total del obispado & 53.100 & 54.600 & 58.975 & 54.975 \\
\hline
\end{tabular}

\begin{tabular}{|l|l|c|c|c|c|}
\hline \multicolumn{7}{|c|}{ Andalucía y Badajoz } \\
\hline 220 & Sevilla & $500^{229}$ & - & - & - \\
\hline 221 & Jerez de la Frontera & 1.000 & - & - & - \\
\hline
\end{tabular}

${ }^{223}$ Con Jaraicejo y Campo de Montánchez (prov. de Cáceres).

${ }^{224}$ Con Jaraicejo (prov. de Cáceres), Orillana y Esparragosa de Lares (prov. de Badajoz).

${ }^{225}$ Idem.

${ }^{226}$ Con Santa Cruz (prov. de Cáceres).

${ }^{227}$ Idem.

${ }^{228}$ Con Garrovillas (prov. de Cáceres).

${ }^{229}$ Con sus ayudas. 


\begin{tabular}{|c|l|c|c|c|c|}
\hline 222 & Sanlúcar de Barrameda & $1.000^{230}$ & - & - & - \\
\hline 223 & Aroche & 500 & - & - & - \\
\hline 224 & Lepe & $500^{231}$ & - & - & - \\
\hline 225 & Moguer & 500 & - & - & - \\
\hline 226 & Córdoba & 500 & $500^{232}$ & - & - \\
\hline 227 & Segura de la Orden & 3.000 & 4.000 & $6.000^{233}$ & $6.500^{234}$ \\
\hline 228 & Llerena & 3.000 & 3.000 & 3.300 & 3.300235 \\
\hline 229 & Fuente del Maestro & $1.000^{236}$ & 1.400 & $2.000^{237}$ & 2.000238 \\
\hline 230 & Fuente de Cantos & 400 & 500 & 500 & 500 \\
\hline 231 & Mérida & 500 & 1.000 & 2.000 & 2.000 \\
\hline 232 & Zafra & $1.500^{239}$ & $2.000^{240}$ & $5.000^{241}$ & $5.400^{242}$ \\
\hline 233 & Jerez de Badajoz & 4.000 & 4.500 & 5.000 & 5.000 \\
\hline 234 & Villanueva de Barcarrota & 1.000 & 1.000 & 500 & 630 \\
\hline 235 & Alconchel & 1.200 & 1.200 & 900 & 1.000 \\
\hline 236 & Burguillos 243 & 2.300 & 2.300 & 2.000 & 2.000 \\
\hline
\end{tabular}

230 Con Rota (prov. de Cádiz).

231 Con Gibraleón (prov. de Huelva).

232 Con Micer Gilo y Bayona (prov. de Córdoba).

233 Con Fuentes (prov. de Badajoz).

234 Idem.

235 Con Guadalcanal (prov. de Sevilla).

236 Con los que con ella pechan.

237 Con Ribera, La Puebla de Sancho Pérez, Los Santos de Maimona, Medina de las Torres y Almendralejo (prov. de Badajoz).

238 Idem.

239 Con La Parra (prov. de Badajoz).

240 Idem.

241 Idem.

242 Idem.

243 C. CARRETE PARRONDO, «Economía de una aljama pacense: Burguillos del Cerro», Actas del IV Congreso Internacional "Encuentro de las Tres Culturas». Toledo 3-7 oct. 1982 (Toledo. 1983) págs. 81-90. 


\begin{tabular}{|c|l|c|c|c|c|}
\hline 237 & Badajoz & $3.000^{244}$ & 3.000 & 2.115 & 2.710 \\
\hline 238 & El Almendral & - & $500^{245}$ & $1.000^{246}$ & $1.000^{247}$ \\
\hline 239 & Villanueva de Fresno & - & - & $500^{248}$ & $500^{249}$ \\
\hline & Total del obispado & 25.400 & 24.900 & 30.815 & 32.540 \\
\hline
\end{tabular}

\section{RESUMEN}

En la sección de Escribanía Mayor de Rentas del Archivo General de Simancas (Valladolid), hemos hallado las listas de los repartimientos que las aljamas y juderías de Castilla realizaron en 1484, 1485, 1490 y 1491, en las que se refleja la cantidad económica que cada una de ellas debía abonar en concepto del impuesto específico para las minorías étnico-religiosas, denominado «servicio y medio servicio», y que consideramos de indudable valor para un mejor y más completo conocimiento de la realidad judía en la España medieval.

\section{SUMMARY}

We have found in the section Escribanía Mayor de Rentas of the Archivo General de Simancas (Valladolid) the annual distribution lists made by the Jewish gatherings and jewries of Castile in 1484, 1485, 1490 and 1491. The texts provide information on the amount they had to pay for the specific tax of the ethnic and religious minorities called «servicio y medio servicio», and thus shed new, important light on Jewish life in medieval Spain.

${ }^{244}$ Con El Almendral (prov. de Badajoz).

${ }^{245}$ Con La Torre (La Torre de Miguel Sesmero) (prov. de Badajoz).

246 Idem.

247 Idem.

248 Con La Higuera (Higuera de Vargas) (prov. de Badajoz).

${ }^{249}$ Idem. 